\title{
Granular contact interfaces with non-circular particles
}

\author{
İlker Temizer* \\ Department of Mechanical Engineering, Bilkent University, 06800 Ankara, Turkey
}

\section{A R T I C L E I N F O}

\section{Article history:}

Received 25 May 2013

Received in revised form

6 August 2013

Accepted 7 August 2013

Available online 17 August 2013

Keywords:

Granular interface

Superellipse

Macroscopic friction

Contact homogenization

\begin{abstract}
A B S T R A C T
The influence of particle geometry on the macroscopic frictional response of granular interfaces is investigated via computational contact homogenization. The particle shape is parametrized by convex superellipse geometries that require iterative closest-point projection schemes for modeling the persistent rolling contact of the particle between a rigid smooth surface and a rubber-like material. Normal and tangential forces acting on the particle are computed by the discrete element method. The non-Amontons and non-Coulomb type macroscopic frictional response of the three-body system is linked to microscopic dissipative mechanisms. Numerical investigations demonstrate rolling resistance and additionally suggest that the macroscopic friction from a complex interface particle geometry may be bound by computations that are based on simplified shapes which geometrically bound the original one.
\end{abstract}

(c) 2013 Elsevier Ltd. All rights reserved.

\section{Introduction}

The microscopic origins of what is measured as friction on the macroscopic scale is largely system dependent. The system might be atomic, molecular or continuum level and a thorough understanding of friction at each level requires addressing experimental, theoretical and computational aspects - see [1] and references therein for recent overviews. The present study lies at the continuum scale where much work has been done within the broader context of tribology [2], constituting a rich basis for modeling and computation.

\subsection{Scope of study}

Consider a macroscopic structure that is made up of rubber in frictional interaction with a smooth surface. It is assumed that the contact interaction is governed locally by an Amontons-Coulomb type friction: the friction coefficient is independent of the contact pressure and slip velocity. It is of interest to determine the effect of introducing particles into the contact interface on the overall interaction of the rubber and the surface. The deformability of both the surface and that of the particles is neglected. For sufficiently small particle sizes, one can imagine that a classical separation of length scales assumption would hold such that the contact interaction is not influenced by the geometrical parameters of the macroscopic structure but rather only by the material properties and the contact variables associated with an infinitesimally thin boundary layer of the rubber in the vicinity of

\footnotetext{
*Tel.: +90 3122903064 .

E-mail address: temizer@bilkent.edu.tr
}

the contact interface. Consequently, it is sufficient to model the microscopic contact interactions only and meld them into a macroscopic friction coefficient $\overline{\mathbb{k}}$ towards a macroscale homogenized interface which has no particles. A computational contact homogenization framework for this purpose has been developed in $[3,4]$. A major observation is that for elastic boundary layers $\overline{\mathbb{k}}$ is explicitly dependent on the macroscopic pressure $\bar{p}$ (nonAmontons). If the boundary layer is additionally viscous then $\overline{\mathbb{k}}$ also depends on the macroscopic frictional slip velocity $\bar{v}_{F}$ (nonCoulomb). In all cases, the macroscopic friction coefficient is a reflection of the microscopic dissipation mechanisms, both at the contact interfaces and within the boundary layer.

Real particles rarely display the idealized circular geometry that has been assumed in the two studies summarized above. The major goal in this contribution is to study the effect of the particle geometry on the macroscopic frictional response of the three-body contact problem described.

\subsection{Flexible particle geometry}

A qualitatively accurate geometrical description of the particle shape remains a challenge in the context of finite element method (FEM) based particulate composite and discrete element method (DEM) based granular material simulations, mainly due to the difficulty in detecting contact among non-spherical geometries. Contact governs the response of granular materials [5] and its detection is intrinsic to the packing of the particles in a simulation domain even if the particles do not physically come into contact but rather constitute a phase in a composite [6]. For composite materials, the underlying FEM discretization allows, in principle, the adoption of arbitrarily complex particle geometries, for instance 
based on spline constructions [7]. When discretization ideas are similarly adopted in DEM [8], contact detection among individual particles with complex shapes benefits from well-known search algorithms in computational contact mechanics [9,10]. Alternative geometries can also be constructed based on approximations, for instance that of an irregular shape by aggregates of spheres or by a polygonal description with flat facets, in effect taking advantage of simple contact detection schemes - see $[11,12]$ for early and more recent overviews as well as for improvements. While further generalizations are possible, e.g. based on computer-aided design tools [13], superellipsoids appear to constitute a reasonable first step in parametrizing non-spherical particles [14,15] and will be adopted here as well.

\subsection{Granular interfaces}

The geometry of the particle determines the contact detection algorithm, not only among the particles but also between a particle and a deformable body. This latter DEM-FEM coupling poses challenges that are case and scale dependent [16]. Presently, the emphasis is on granular interfaces [17-19]. Typically, such interfaces naturally occur with a large number of particles such that the macroscopic response is of the lubrication type [20,21]. See [22] for a recent comprehensive review of interfaces with third bodies.

One distinguishing feature of the present contribution is that it concentrates on the (i) persistent and (ii) large sliding contact of a non-circular particle with (iii) an elastomeric material that deforms significantly. Consequently, the FEM elements do not simply constitute obstacles with which the DEM particles come into contact and exchange momentum - cf. [23,24]. The mesh and the time discretization should be fine enough to capture the microscale interactions. Roughly, this translates into the requirements that (i) the mesh is able to deform around and encapsulate the particle at the contact interface, and also that (ii) the FEM system is solved at each DEM time step. These are factors that together contribute to a high computational cost.

\subsection{Limitations}

There are two major shortcomings with the present framework. First, within the two-dimensional setting of the present study the rubber-particle interactions are too stiff in the sense that, for a given fraction of surface coverage by the particles, the rubber cannot as easily encapsulate the particle as in the three-dimensional setting. Consequently, for a realistic choice of material parameters for the rubber, the magnitude of the macroscopic contact pressure needed to initiate rubber-surface contact beyond rubber-particle contact will be unrealistically high. Moreover, the possibly complex dynamics of a threedimensional non-spherical particle at the interface cannot be captured. However, considering that the simulation time for a single run in a parametrical study easily exceeds an hour on a standard workstation, the dimensional simplification is greatly facilitating. Second, even without the presence of the particles at the contact interface, rubber friction is known to be already of a non-Amontons and non-Coulomb type behavior [25]. The complex tribological behavior of rubber has long been a subject of experimental interest [26,27] and it was recognized early that this behavior is governed by the interaction of rubber with micro-rough surfaces through viscoelastic and adhesive mechanisms [28] - see [29] for a more recent study. Recent theoretical and computational studies have greatly added to the understanding of rubber friction [30-35]. Hence, even a qualitative comparison with experimental results would require enhancing the framework with rubber-surface contact models that are more realistic than the constant friction coefficient assumption employed presently.

Nevertheless, the developed framework is still capable of making qualitative predictions regarding the effect of the particle presence at the contact interface, in particular with respect to the particle shape. Recent experimental studies on the types of granular interfaces considered demonstrated that the presence of the particles leads to a reduction in the macroscopic friction coefficient in comparison with the raw interface [36] and that a strong pressure dependence is observed [37]. While a direct comparison with experimental results is outside the scope of the present study, it is noted that these observations are encouraging since they are qualitatively in line with the numerical predictions. A three-dimensional framework with enhanced friction models as well as an experimental programme is currently under investigation.

\subsection{Outline}

The remainder of this work is organized as follows. In Section 2, the problem setting is summarized and the major simulation parameters are introduced. Aspects of dealing with non-circular particles, in particular contact detection and force computation schemes, are discussed in Section 3. The finite nonlinear viscoelasticity model employed in earlier studies is briefly outlined in Section 4 in order to introduce the extension for including the Payne effect as an important filled rubber behavior. Representative numerical examples and parametric studies are provided in Section 5.

A self-contained presentation is pursued only to the extent that is relevant to the novel aspects introduced. Various technical details on material modeling, DEM aspects for the particles, FEM aspects for contact mechanics, DEM-FEM coupling and other computational issues regarding contact homogenization which have previously been addressed in [3,4] are omitted for brevity.

\section{Micromechanical test}

The micromechanical test setup depicted in Fig. 1 will be employed in order to extract the macroscopic frictional response of the granular interface. Major simulation parameters are summarized in Table 1. Unless stated otherwise, these values will be employed in all numerical examples.

\subsection{Test setup}

Within the two-phase micromechanical test, a rubber block $\mathcal{C}_{0}$ with dimensions $H_{O} \times L_{O}$ is first pressed against the granular surface by enforcing the macroscopic pressure $\bar{p}$ (compression phase) and subsequently dragged at the macroscopic slip velocity $\bar{v}_{F}$ while maintaining the applied macroscopic pressure (dragging phase). The raw friction coefficient for the rubber-surface interface is denoted by $\mathbb{k}_{0}$ while those at the particle-surface and the particle-rubber interfaces are denoted by $\mathbb{k}_{P S}$ and $\mathbb{k}_{P B}$ respectively. All friction coefficients are assumed to be independent of the local contact pressure and slip velocity.

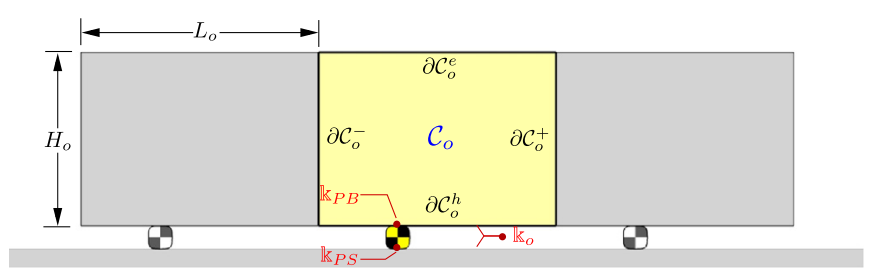

Fig. 1. The mesoscopic test setup geometry with a non-circular particle. 
Table 1

Default simulation parameters. Only the changes to these default choices will be explicitly noted.

\begin{tabular}{lll}
\hline Test sample length (mm) & $L_{o}$ & 10 \\
Test sample height $(\mathrm{mm})$ & $H_{o}$ & 7.5 \\
Reference radius $(\mathrm{mm})$ & $r_{o}$ & 0.5 \\
Macroscopic contact pressure (bar) & $\bar{p}$ & (Varied) \\
Macroscopic slip velocity (m/s) & $\bar{v}_{F}$ & 2.5 \\
Elastic shear modulus $(\mathrm{MPa})$ & $\mu_{e}$ & 1 \\
Particle density $\left(\mathrm{g} / \mathrm{cm}^{3}\right)$ & $\rho_{P}$ & 3 \\
Nominal dragging time & $T_{o}$ & $L_{o} / \bar{v}_{F}$ \\
Total averaging time & $T_{\mathrm{avg}}$ & $2 T_{o}$ \\
Nominal time step size & $\Delta t$ & $10^{-4} T_{o}$ \\
Friction coefficient & $\mathbb{k}_{o}$ & 0.5 \\
& $\mathbb{k}_{\mathrm{k} P}$ & 0.5 \\
Mesh resolution & $\mathbb{k}_{\mathrm{kS}}$ & 1.5 \\
& $N_{X}$ & 40 \\
Penalty parameter & $N_{Y}$ & 15 \\
& $\epsilon_{N}$ & $10^{6} \mu_{e}$ \\
& $\epsilon_{T}$ & $10^{4} \mu_{e}$ \\
\hline
\end{tabular}

In order to handle particles leaving the contact zone during the dragging phase, periodicity conditions are assumed, complemented by periodic boundary conditions on $\partial \mathcal{C}_{o}^{p}:=\partial \mathcal{C}_{o}^{-} \cup \partial \mathcal{C}_{o}^{+}$:

$\boldsymbol{u}^{+}=\boldsymbol{u}^{-}$and $\boldsymbol{t}^{+}=-\boldsymbol{t}^{-}$on $\partial \mathcal{C}_{0}^{p}$.

Here, $\boldsymbol{u}$ is the displacement vector and $\boldsymbol{t}$ is the Cauchy traction. Consequently, there are image cells on both sides of the central cell although the computation is carried out explicitly within the central cell only. Only a single particle is assumed to be present due to the assumption of a periodic particle distribution. The surface coverage of the particles is controlled by the length of the sample and also by the size of a particle for a fixed length. Presently, the geometry of the sample will be fixed and only the particle shape will be varied.

\subsection{Simulation parameters}

The rubber block is discretized with $N_{X} \times N_{Y}$ elements. In order to enforce incompressibility, the material model for the rubber is embedded within a $Q 1 P 0$ formulation. The elastic response of rubber can be accurately described through an Ogden-type constitutive formulation [38] with an elastic shear modulus $\mu_{e}$ and Ogden material parameters used ${ }^{1}$ in [4]. The elastic bulk modulus is chosen to be $\kappa_{e}=10^{3} \mu_{e}$ such that the material is nearly incompressible. The inertia of the rubber is neglected. However, it is numerically advantageous to benefit from the regularizing effects of dynamics, in particular with respect to accelerating the convergence of the contact active set. For this purpose, the density of the rubber block is assigned a small value $\rho_{B}=10^{-3} \rho_{P}$ where $\rho_{P}$ is the density of a particle. The inertia of the particle, on the other hand, is important from a DEM perspective. When the particle shape is varied, the mass and the moment of inertia will change. The aim of the present work is to isolate the effect of the particle shape on the macroscopic frictional response. Consequently, the particles will always be assigned the mass and the moment of inertia of a reference circular particle with a fixed radius $r_{o}$.

\subsection{Microscopic and macroscopic contact interactions}

At the particle-rubber and rubber-surface interfaces, the classical penalty regularization is employed to enforce the contact constraints using normal $\left(\epsilon_{N}\right)$ and tangential $\left(\epsilon_{T}\right)$ penalty parameters. The constraints are enforced at the two integration points

\footnotetext{
${ }^{1}$ There is a typo in Table 1 of this reference: $\gamma_{2}$ should be -0.231 .
}

of each linear contact element. This formulation does not deliver optimal local contact interactions (cf. mortar-based approaches [39-42]) although the macroscopic normal $\left(F_{N}\right)$ and tangential $\left(F_{T}\right)$ forces applied to the sample, measured at the observable test surface $\partial \mathcal{C}_{o}^{e}$ (Fig. 1), are predicted to good accuracy provided the penalty parameters are chosen judiciously. The macroscopic friction coefficient during sliding is then instantaneously measured by

$\overline{\mathbb{k}}_{I}=\left|\frac{F_{T}}{F_{N}}\right|$.

Due to dynamic effects such as the particle-rubber interaction, $\bar{k}_{I}$ is highly oscillatory in time, in particular during the transition from compression $\left(0<t<T_{o}\right)$ to dragging $\left(t>T_{o}\right)$. For this reason, the moving time average $\overline{\mathbb{K}}_{I}$ of $\overline{\mathbb{k}}_{I}$ is monitored for convergence after the transition region $\left(T_{o}<t<2 T_{o}\right)$, the total duration of which is $T_{\text {avg }}$ seconds. The value at convergence is designated as the macroscopic friction coefficient $\bar{k}$ of the setup. This is the value which will be reported throughout the investigations.

\section{Non-circular particles}

\subsection{Superellipse geometry}

Following earlier approaches for particulate composite modeling and DEM, deviations from an idealized circular shape can be parametrized by a superellipse

$\sum_{i}\left(\frac{\left|\boldsymbol{d}_{i} \cdot(\boldsymbol{x}-\boldsymbol{c})\right|}{r_{i}}\right)^{p_{i}}-1=0$

where $\boldsymbol{c}$ is the center of the particle, $\boldsymbol{d}_{i}$ are principal axis orientations, $r_{i}$ are principal radii and $p_{i}$ control the particle shape. In particular, $p_{i} \geq 2$ ensures the convexity of the particle surface, which is a necessary ingredient in the Newton-Raphson method to be employed in detecting particle-surface and particle-rubber contact. For contact detection, it is advantageous to employ a local coordinate system defined by a convected basis $\left\{\boldsymbol{d}_{1}, \boldsymbol{d}_{2}\right\}$ such that, using $\boldsymbol{x}-\boldsymbol{c}=\boldsymbol{y}=y_{i} \boldsymbol{d}_{i}$, at a given time a point $\mathcal{P}$ on the particle surface satisfies (see Fig. 2)

$f(\boldsymbol{y})=\sum_{i}\left(\frac{\left|y_{i}\right|}{r_{i}}\right)^{p_{i}}-1=0$.

In all subsequent discussions, the vector components are algorithmically evaluated with respect to the $\boldsymbol{d}$-basis, using the particle center as the origin for position vectors. Mapping between the global $\boldsymbol{e}$-basis and the local $\boldsymbol{d}$-basis is accomplished through standard rotation and translation operations associated with the particle motion.

\subsection{Particle-rubber contact}

Particle-rubber contact detection is based on the classical closest-point projection algorithm. The outward unit normal $\boldsymbol{n}$ to the particle surface is obtained by $\boldsymbol{n}=\nabla f /\|\nabla f\|$ which defines the tangent vector $\boldsymbol{a}_{T}=\boldsymbol{e}_{3} \times \boldsymbol{n}$. The closest-point projection $\boldsymbol{y}_{p}$ of a contact element integration point $\boldsymbol{m}$ is defined by the requirement

$\boldsymbol{a}_{T} \cdot\left(\boldsymbol{m}-\boldsymbol{y}_{p}\right)=0$,

subject to the constraint

$f\left(\boldsymbol{y}_{p}\right)=0$.

In order to solve for $\boldsymbol{y}_{p}$, all calculations are performed in the first quadrant of the particle. Within the Newton-Raphson method for determining $\boldsymbol{y}_{p}$, it is advantageous to iterate in a specific vector component to avoid potential ill-conditioning. The component is chosen depending on the position of the projected point with 

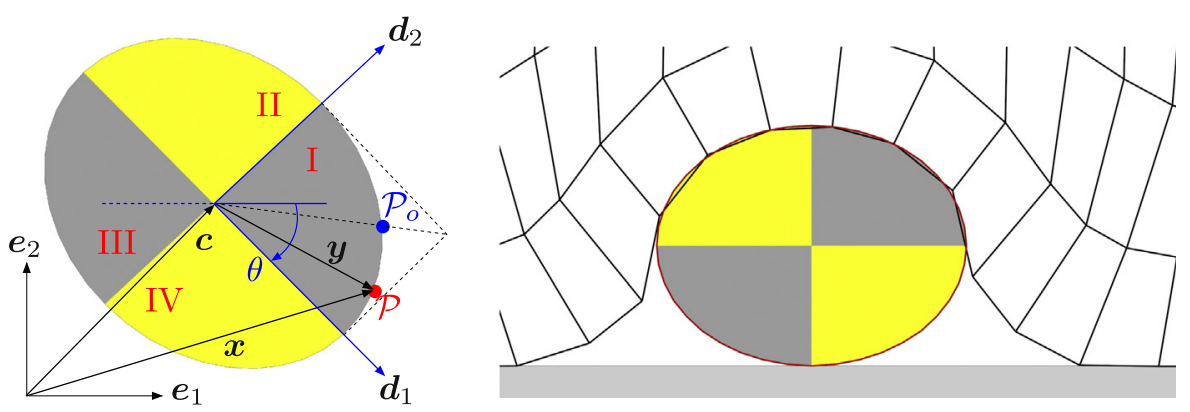

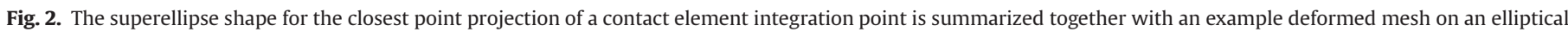
particle. Here, $a_{r}=1.25$ and $p_{1}=p_{2}=2$. The normal to the plane is given by $\boldsymbol{e}_{3}=\boldsymbol{e}_{1} \times \boldsymbol{e}_{2}$.

Table 2

Algorithm for the closest point projection of a mesh point $\boldsymbol{m}$ onto a superellipse.

1. Contact check. Proceed with projection if $f(\boldsymbol{m})<0$.

2. Quadrant check. Record the quadrant of projection: $q_{i}=\left|m_{i}\right| / m_{i}$

3. Constraint elimination. Map $\boldsymbol{m}$ to its first quadrant image $\boldsymbol{m}^{\prime}=|\boldsymbol{m}|$ and eliminate the constraint on the image $y_{p i}^{\prime}=\left|y_{p i}\right|$ of the projection point via

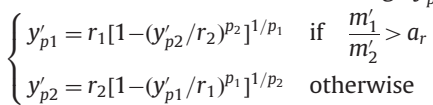

4. Projection. Determine $\boldsymbol{a}_{T}\left(\boldsymbol{y}_{p}^{\prime}\right) \perp \boldsymbol{n}\left(\boldsymbol{y}_{p}^{\prime}\right) \|\left.\nabla f\right|_{\boldsymbol{y}_{p}^{\prime}}$ and solve for $y_{p 1}^{\prime}$ or $y_{p 2}^{\prime}$ from the nonlinear orthogonality relation of closest point projection: $\boldsymbol{a}_{T} \cdot\left(\boldsymbol{m}^{\prime}-\boldsymbol{y}_{p}^{\prime}\right)=0$

5. Quadrant map. Map to the quadrant of physical contact: $y_{p i}=q_{i} y_{p i}^{\prime}$

respect to a point $\mathcal{P}_{o}$ on the particle (Fig. 2) with the property $y_{1}=a_{r} y_{2}$ where

$a_{r}=\frac{r_{1}}{r_{2}}$

is the aspect ratio. The point $\mathcal{P}_{0}$ is fixed for given $\left\{r_{i}, p_{i}\right\}$ and is determined in a pre-processing step. The algorithm for determining $\boldsymbol{y}_{p}$ requires straightforward calculations and is summarized in Table 2. After its determination, it is mapped to the physical position $\boldsymbol{x}_{p}$ which is then passed back to the contact element for penalization. Fig. 2 displays an example deformed mesh on an elliptical particle.

\subsection{Particle-surface contact}

The particle-surface contact algorithm has two ingredients: (i) determination of the point of potential contact $\boldsymbol{y}_{c}$ between the particle and the surface and (ii) evaluation of the normal and tangential contact forces $\left\{f_{N}, f_{T}\right\}$ acting on the particle. Although an analytical solution was previously employed for the latter purpose with circular particles, the non-circular particle shape requires a more general contact model.

\subsubsection{Determination of the contact point}

The point of potential contact $\boldsymbol{y}_{c}$ corresponds to the closestpoint projection of the particle to the surface if there is no contact and to the maximum penetration point otherwise. The slope $s=\mathrm{d} y_{c 2} / \mathrm{d} y_{c 1}$ at this point is zero. Therefore, its computation is easily carried out by first determining the actual quadrant of the contact point and then the slope $s^{\prime}=\mathrm{d} y_{c 2}^{\prime} / \mathrm{d} y_{c 1}^{\prime}$ at the image $\boldsymbol{y}_{c}^{\prime}$ of this point with respect to the $\boldsymbol{d}$-basis in the first quadrant where calculations are carried out. Based on the clockwise angle of a

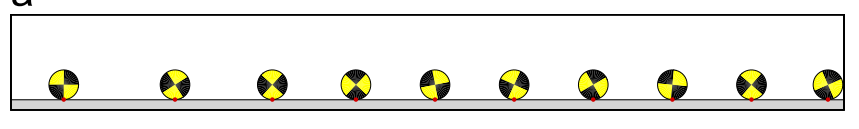

$a_{r}=1, p_{1}=p_{2}=2($ CIRCULAR $)$

b

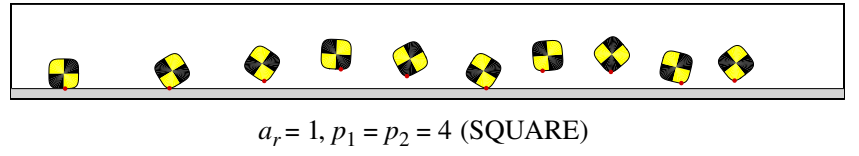

C

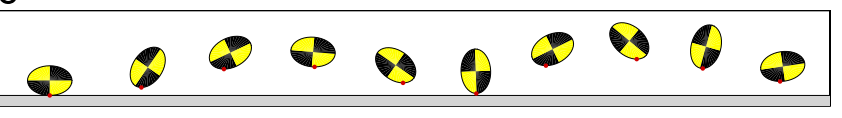

$a_{r}=1.5, p_{1}=p_{2}=2($ ELLIPTICAL $)$

d

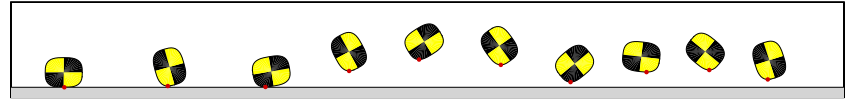

$a_{r}=1.25, p_{1}=p_{2}=3($ RECTANGULAR)

Fig. 3. The potential contact point $(\bullet)$ between the particle and the surface is shown for various instances. In all simulations, the particle is initially assigned a horizontal velocity. The circular particle first slides and subsequently makes a transition to rolling without slipping. A non-circular particle $\left(r_{2}=r_{o}\right)$ impacts the surface and bounces off during rolling due to its irregular shape. The geometries displayed above will be employed in all upcoming numerical investigations as well.

particle rotation $\theta$ (Fig. 2), these are

QuAdRANT : $\left\{\begin{array}{lll}\text { I } & \text { if } \cos \theta<0, & \sin \theta \geq 0 \rightarrow s^{\prime}=-\tan \left(180^{\circ}-\theta\right) \\ \text { II } & \text { if } \cos \theta<0, & \sin \theta<0 \rightarrow s^{\prime}=-\tan \left(\theta-180^{\circ}\right) \\ \text { III } \quad \text { if } \cos \theta \geq 0, & \sin \theta<0 \rightarrow s^{\prime}=-\tan \left(360^{\circ}-\theta\right) \\ \text { IV } \quad \text { if } \cos \theta \geq 0, & \sin \theta \geq 0 \rightarrow s^{\prime}=-\tan (\theta)\end{array}\right.$

Within the Newton-Raphson method for determining the image point $\boldsymbol{y}_{c}^{\prime}$ on the particle where the slope is $s^{\prime}$, it is advantageous to iterate in $y_{c 1}^{\prime}$ if $\left|s^{\prime}\right|<\left|s_{o}\right|$ and in $y_{c 2}^{\prime}$ otherwise, where $s_{o}$ is the reference value at $\mathcal{P}_{o}$ (Fig. 2). Once the components $y_{c i}^{\prime}$ are determined, they are first mapped back to $y_{i c}=q_{i} y_{c i}^{\prime}$, e.g. $q_{1}=-1$ and $q_{2}=1$ in quadrant II, and subsequently to the physical position $\boldsymbol{x}_{c}$ of the contact point. Simulation instances displaying the point of potential contact are shown in Fig. 3 for particles with different shapes.

\subsubsection{Determination of the contact forces}

The contact force acting on a particle is $\boldsymbol{f}=f_{T} \boldsymbol{e}_{1}+f_{N} \boldsymbol{e}_{2}$. If $\Delta \boldsymbol{x}_{C}$ is the relative change in the position of the material point associated with $\boldsymbol{x}_{c}$ through a time step $\Delta t$, assuming that the contact surface is at $x_{2}=0$, normal and tangential kinematic contact variables are 
defined by

$d_{N}=-x_{c 2}, \quad d_{T}^{\text {trial }}=d_{T}^{\text {old }}+\Delta x_{c 1}$

such that $d_{N}>0$ indicates contact and $d_{T}^{\text {old }}$ plays the role of a history variable.

In DEM, the normal force is typically determined based on viscoelastic Hertzian contact under the constraint that the interface cannot support tension [5]

$f_{N}=\max \left(0, k_{N} \sqrt{d_{N}}\left[d_{N}+c_{N} \dot{d}_{N}\right]\right)$.

The viscous contribution is responsible for the energy loss during impact and as such can be related to the coefficient of restitution [43]. Although it is not essential to the purposes of the present study, the viscous contribution was observed to help damp out oscillations in the instantaneous macroscopic friction coefficient $\overline{\mathbb{k}}_{I}$ that appear in its absence during the persistent rolling contact of the particle between the rubber and the surface. However, although damping during compression $\left(\dot{d}_{N} \geq 0\right)$ is favorable as such, it was also observed that the classical formulation above can lead to a premature loss of contact between the particle and the surface during decompression $\left(\dot{d}_{N}<0\right)$ due to the negative viscous contribution to the normal force. A premature loss of contact leads to an unrealistic slip detection and, hence, to an incorrect macroscopic friction coefficient prediction. Therefore, the formulation above is employed by deactivating damping during decompression. In all simulations, gravity is additionally included.

The tangential force is modeled via a penalty regularization together with an Amontons-Coulomb friction based on the stick

Table 3

Numerical values of the default DEM parameters.

\begin{tabular}{lll}
\hline Normal stiffness & $k_{N}$ & $10^{5} \mu_{e}$ \\
Tangential stiffness & $k_{T}$ & $10^{3} \mu_{e}$ \\
Normal viscosity & $c_{N}$ & $3 \times 10^{-5}$ \\
\hline
\end{tabular}

\section{a}

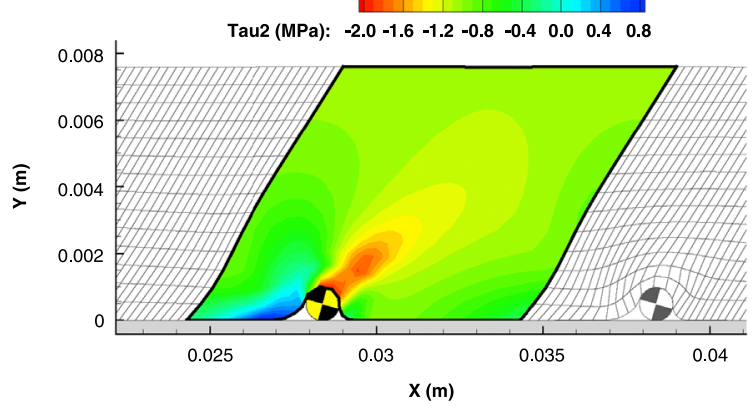

C

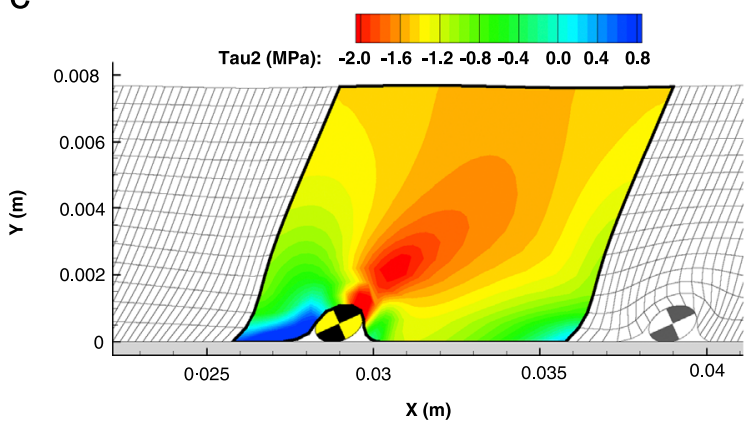

prediction

$f_{T}^{\text {trial }}=-k_{T} d_{T}^{\text {trial }}$

that is corrected if slip is detected:

$f_{T}=\left\{\begin{array}{l}f_{T}^{\text {trial }} \text { and } \quad d_{T}=d_{T}^{\text {trial }} \quad \text { if }\left|f_{T}^{\text {trial }}\right|-\mathbb{k}_{P S} f_{N} \leq 0 \text { (STICK) } \\ \mathbb{k}_{P S} f_{N} \frac{f_{T}^{\text {trial }}}{\left|f_{T}^{\text {trial }}\right|} \text { and } d_{T}=-\frac{f_{T}}{k_{T}} \text { otherwise (SLIP) }\end{array}\right.$

The variable $d_{T}$ is reset to zero whenever there is contact loss.

The constants appearing in the normal and tangential contact models can be obtained from the material properties of the particle [5]. Presently, the viscous constant is chosen so as to obtain sufficient damping. The elastic constants, on the other hand, are chosen to satisfy the conflicting requirements of (i) minimal penetration and (ii) maximal time step size. The latter is particularly important since a linear system of equations emanating from the FEM model for rubber must be solved at each time step. Default choices employed are summarized in Table 3. Fig. 3 simulation instances were based on this set of parameters. These choices lead to reasonable simulation times $\left(\Delta t=10^{-4} T_{o}-\right.$ see Table 1 ) and also deliver satisfactorily small penetrations under the significantly (compared to Fig. 3) large forces applied during persistent rolling contact with the rubber. Coupled FEMDEM simulation instances from the dragging phase with various particle shapes are provided in Fig. 4.

\section{Viscoelastic boundary layers}

\subsection{Dissipation mechanisms}

Viscoelastic boundary layers continuously dissipate energy due to the cyclic loading of the rubber by the particle during dragging. This dissipation augments the macroscopic friction coefficient, which is essentially a parameter that reflects the lumped effect of all microscopic dissipation mechanisms. Other inelastic

b

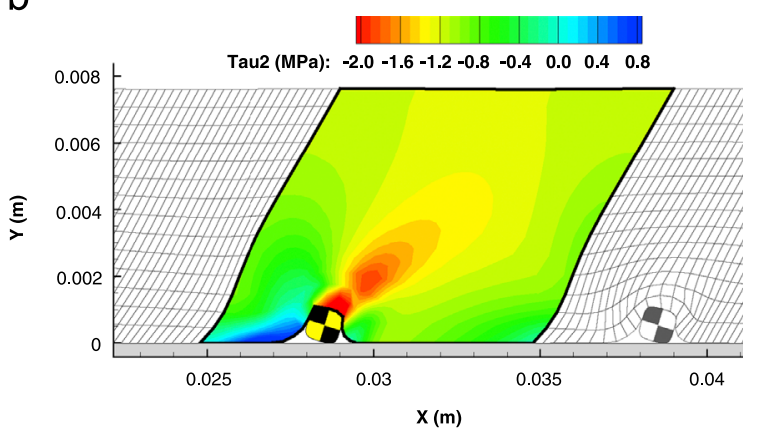

d

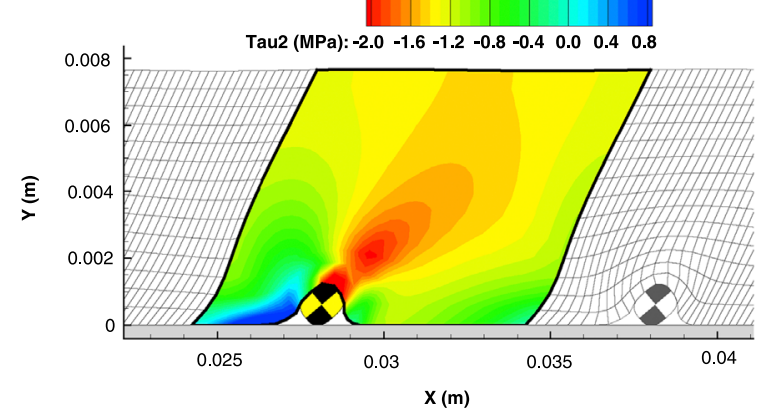

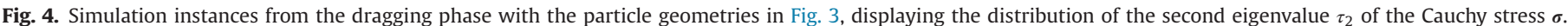
During compression $\tau_{2} \approx-\bar{p}$ ( $=-2 \mathrm{MPa}$ ) on $\partial \mathcal{C}_{o}^{e}$ (see Fig. 1 ), making it a convenient quantity for monitoring. (a) Circular, (b) square, (c) elliptical, and (d) rectangular. 
mechanisms, such as damage reflecting the Mullins effect at large deformations, also lead to dissipation during the initial phase of dragging. However, rate-independent mechanisms significantly saturate after a few cycles of loading. Consequently, at steady state, their effect can be reflected by a composite boundary layer with modified elastic properties in the immediate vicinity of the surface. Even then, viscoelastic effects are significantly more dominant [4]. For this reason, damage mechanisms will be omitted in the present study. Hence, recalling that adhesive effects are also neglected, the macroscopic friction coefficient may be additively decomposed into frictional $\left(\overline{\mathbb{k}}_{F}\right)$ and viscous $\left(\overline{\mathbb{k}}_{V}\right)$ contributions from microscopic dissipation mechanisms:

$\overline{\mathbb{k}}=\overline{\mathbb{k}}_{F}+\overline{\mathbb{k}}_{V}$

In the absence of viscous dissipation, $\overline{\mathbb{k}}_{F}$ still differs significantly from the raw friction coefficient $\mathbb{k}_{0}$. In particular, it varies with the macroscopic pressure but not with the macroscopic slip velocity. The viscous contribution additionally renders the macroscopic response dependent on the macroscopic slip velocity.

\subsection{Viscoelasticity model}

In order to incorporate viscous effects, the standard (non)linear solid model is employed where a hyperelastic branch is in parallel with a viscous Maxwell branch. Here, the constitutive model for the Maxwell branch is constructed based on the multiplicative decomposition of the deformation gradient to elastic and viscous parts. The evolution of the viscous part is constructed to obtain positive dissipation for thermodynamic consistency - see [44] for details. Such consistency is crucial to ensure that the viscous contribution $\bar{k}_{V}$ is non-negative. This model inherits the classical linear viscoelasticity parameters. Consequently, the three core variables are the shear moduli of the elastic $\left(\mu_{e}\right)$ and viscous $\left(\mu_{v}\right)$ branches as well as the shear relaxation time $\tau_{o}=\eta_{o} / \mu_{v}$ of the viscous branch with $\eta_{0}$ as the viscosity. The model only affects the deviatoric stress. There are no bulk contributions from the viscous branch.

For a fixed loading frequency in the kinematically linear regime, a single $\left\{\mu_{v}, \tau_{0}\right\}$ combination can reflect the effect of all viscous mechanisms since the storage and loss moduli at a fixed frequency are functions of these two variables only. However, for the loading scenarios of the present study where the slip velocity varies, nonlinear deformations are sustained and the rubber experiences relaxation periods in between particle excitations, ideally multiple Maxwell branches are required to accurately reflect the dependence on the macroscopic slip velocity. Nevertheless, a single Maxwell branch is employed to capture the qualitative effect of viscoelasticity. The default relaxation time is chosen according to the representative time scale associated with the loading of the sample by the particle as it rolls across the cell boundaries, which is roughly of order $L_{o} / \bar{v}_{F}$.

\subsection{Payne effect}

For the standard Maxwell element, the storage and loss moduli at a fixed frequency do not depend on the amplitude of loading. This is a satisfactory approach for the modeling of unfilled rubber. For filled rubber that is often used in tires, however, this contradicts with the Payne effect where the storage modulus rapidly decreases while the loss modulus first increases and then decreases for increasing deformations about an equilibrium state [45]. An example calculation displaying its influence is shown in Fig. 5. The Payne effect occurs predominantly at small deformations, it is rate-dependent, it affects the viscous mechanisms and is reversible. This should be compared with the Mullins effect mentioned in Section 4.1: it occurs at large deformations, it is often modeled as rate-independent, it predominantly affects the elastic mechanisms and is not reversible. The Payne effect appears not to have been considered in multiscale rubber friction models earlier. Considering its direct influence on the viscosity and its reversibility, it is expected to have a significant influence on the macroscopic frictional response.

In order to capture the Payne effect in the finite deformation regime, the model proposed recently in [45] is employed. At a fixed frequency of loading, this model satisfactorily captures the storage and loss moduli evolution with the loading amplitude, even with a single Maxwell branch. For this purpose, the actual viscosity $\eta=\tau \mu_{v}$ of the Maxwell branch is proposed in the form:

$\eta=\frac{\eta_{0}}{H} \longleftarrow H(t)=1+d q(t)$

where the evolution law for $q(t)$ is

$\dot{q}=\frac{1}{\lambda}(\|\boldsymbol{D}\|-q)$

with $\boldsymbol{D}$ as the symmetric part of the velocity gradient. If $d=0$ then there is no Payne effect. Otherwise, at a fixed amplitude of a constant frequency loading, $q$ (initially zero) evolves towards an equilibrium value at a rate that is controlled by the relaxation parameter $\lambda$. Default values of the viscoelasticity parameters are summarized in Table 4.

\section{Numerical investigations}

In this section, the effect of the particle geometry on the macroscopic frictional response and how this effect interacts with the material behavior will be demonstrated. Different geometries are used to highlight a series of observations, always in comparison with the response based on a circular particle. In reference to [3], it is noted that all simulations will be carried out using an explicit DEM-FEM coupling strategy. In order to update the particle position from time $t_{n}$ to $t_{n+1}$, an implicit approach attempts to make use of the force and the moment acting on the particle at $t_{n+1}$, hence requiring iterations within a time step, while an explicit approach makes use of the force and the moment from $t_{n}$. An implicit approach was found favorable with circular particles in order to enable the use of larger time steps. However, the time step size required for non-circular particles is already sufficiently small for a satisfactory use of the explicit scheme.

\subsection{Elastic and viscous effects}

As for a circular particle, non-circular particles lead to a macroscopic friction coefficient $\overline{\mathbb{k}}$ that strongly depends on the macroscopic contact pressure $\bar{p}$. Fig. 6(a) displays both this dependence as well as the significant effect of the particle geometry for a purely elastic material response within the boundary layer. When a viscoelastic response is employed, two competing effects come into play: (i) the stiffness of the material increases so that the rubber cannot as easily encapsulate the particle, which contributes to a decreasing $\overline{\mathbb{k}}_{F}$, while (ii) the viscous dissipation contributes to the macroscopic friction through $\overline{\mathbb{}}_{V}$. With the default simulation parameters, the viscous contribution dominates such that $\bar{k}$ is higher than the one for a purely elastic response. This is demonstrated in Fig. 6(b) for an elliptical particle. The Payne effect spans the range between the elastic and viscoelastic responses with a varying magnitude of the parameter $d$ in (14) which controls the degree of reduction in the viscosity $\eta$. If $d$ is too small, the response is closer to the viscoelastic response without the Payne effect whereas if it is relatively large then the reduction in $\eta$ is sufficiently high so as to entirely deactivate the contribution from the Maxwell branch. 
a

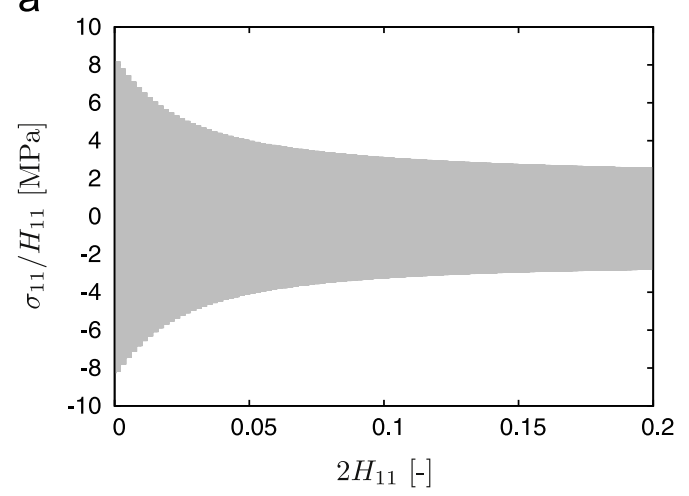

C

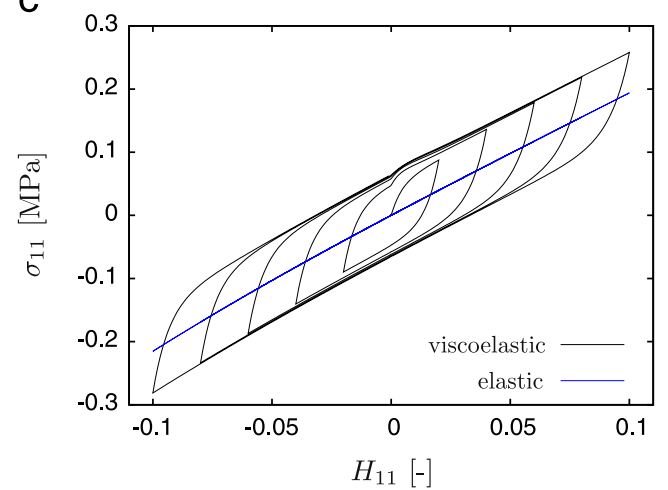

b

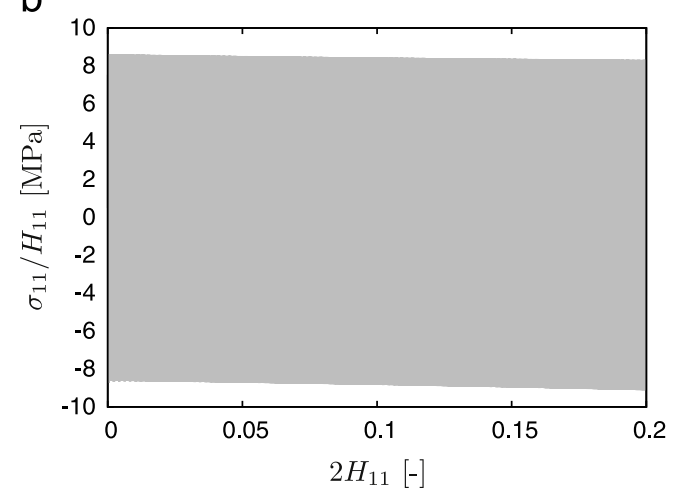

d

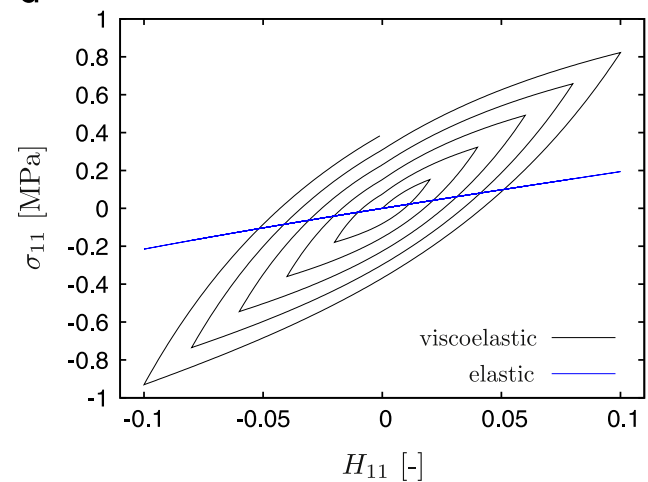

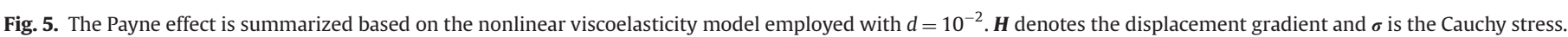

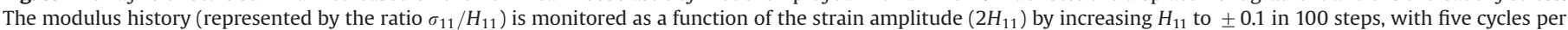

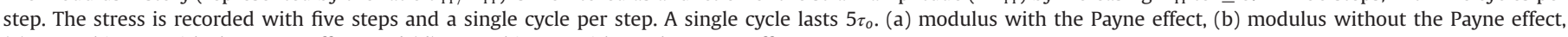
(c) stress history with the Payne effect, and (d) stress history without the Payne effect.

Table 4

Default viscoelasticity parameters that supplement Table 1.

\begin{tabular}{lll}
\hline Shear modulus & $\mu_{v}$ & $5 \mu_{e}$ \\
Relaxation time (s) & $\tau_{0}$ & $10^{-4}$ \\
Payne effect (s) & $d$ & (Varied) \\
& $\lambda$ & $0.1 \tau_{o}$ \\
\hline
\end{tabular}

Despite the significantly non-circular geometry, the variation of the frictional $\left(\overline{\mathbb{k}}_{F}\right)$ and viscous $\left(\overline{\mathbb{K}}_{V}\right)$ contributions to $\overline{\mathbb{k}}_{k}$ with $\bar{p}$ is qualitatively identical to a circular one, as summarized in Fig. 7 for the rectangular particle. Other particle geometries induce a similar behavior. It is also observed that $\overline{\mathbb{k}}_{F}$ approximately retains its value from the purely elastic response so that the overall change in $\overline{\mathbb{k}}$ is due to the additional contribution $\overline{\mathbb{k}}_{V}$. It is noted that, in the given range of simulation parameters, the particles always displayed a rolling motion at the particle-surface interface. Consequently, the macroscopic friction coefficient did not exceed the raw value $\mathbb{k}_{0}$ in any of the test cases even for the non-circular particles.

The instantaneous macroscopic frictional response also strongly depends on the particle geometry, as demonstrated in Fig. 8. The smooth rolling motion of a circular particle causes relatively minor oscillations. Non-circular particles tend to rest on the longer or flat side with no slip so that tangential force starts to build on the particle. It then, relatively rapidly, rolls over the sharp edge until rolling stops once again. These sudden transitions between two rest states cause stronger oscillations in the instantaneous macroscopic friction and essentially imply a rolling resistance. It is remarked that the time step size is crucial to properly resolving the interface dynamics. If not properly chosen, the rolling motion of the particle may be completely or partially arrested. Under a viscoelastic material response, the oscillations can significantly increase, although the transitions are somewhat smoothened. In order to ensure a convergent time-averaged macroscopic response $\overline{\mathbb{k}}_{I}$ in the presence of such oscillations, the averaging time $T_{\text {avg }}$ was chosen to be twice as high as the original choice $2 T_{o}$ - see Section 2.3.

\subsection{Frequencies of excitation}

As the macroscopic slip velocity $\bar{v}_{F}$ is varied, the frequency of excitation of the viscoelastic boundary layer by the particle changes. For sufficiently low slip velocities the material is always in a relaxed state while for high slip velocities the material does not relax at all. Consequently, intermediate slip velocities deliver a macroscopic frictional response that converges to these two extremes. Rather than varying the slip velocity directly, one can also scale the relaxation time since the equivalent relaxation time $\tau_{o} \times \bar{v}_{F}$ is expected to control the macroscopic response [4,35]. This is carried out in Fig. 9 by omitting the Payne effect. For a circular particle, a response that is typical of rubber friction is observed where the transition from the full- to the zero-relaxation limit is smooth. For this example, a stationary particle would move through the unitcell at a period $L_{o} / \bar{v}_{F}=4 \times 10^{-3}$ which is close to where the viscous dissipation, and hence the viscous contribution $\overline{\mathbb{k}}_{V}$ to $\bar{k}$, peaks. For a square particle, it is observed that the viscous contribution makes not one but rather two peaks. The lower period, or the higher frequency, associated with the first peak is due to the rapid rolling of the non-circular particle over its edges. The angular velocity of a particle is of order $\omega \approx \bar{v}_{F} / 2 r_{o}$ so that the period of this motion is of order $\pi / 2 \omega<10^{-3}$, which is in the vicinity of the observed peak. The additional dissipation due to this high frequency excitation 

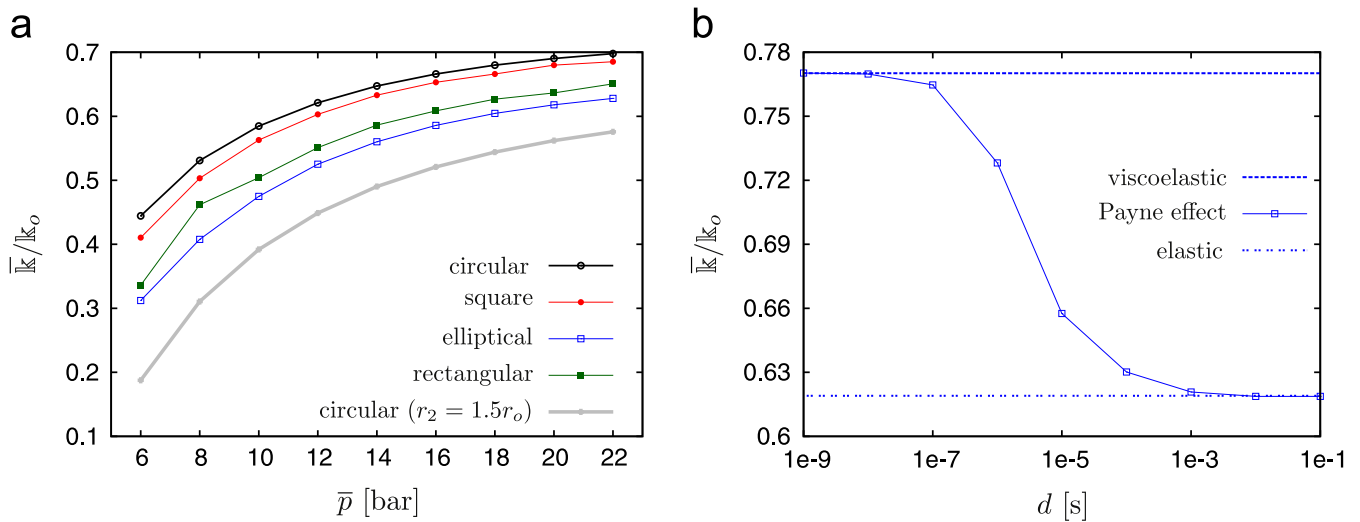

Fig. 6. The effects of (a) the particle shape and (b) viscoelasticity with the Payne effect are demonstrated. See Fig. 3 for the particle geometry parameters. (a) Elastic response and (b) viscoelastic response - elliptical.
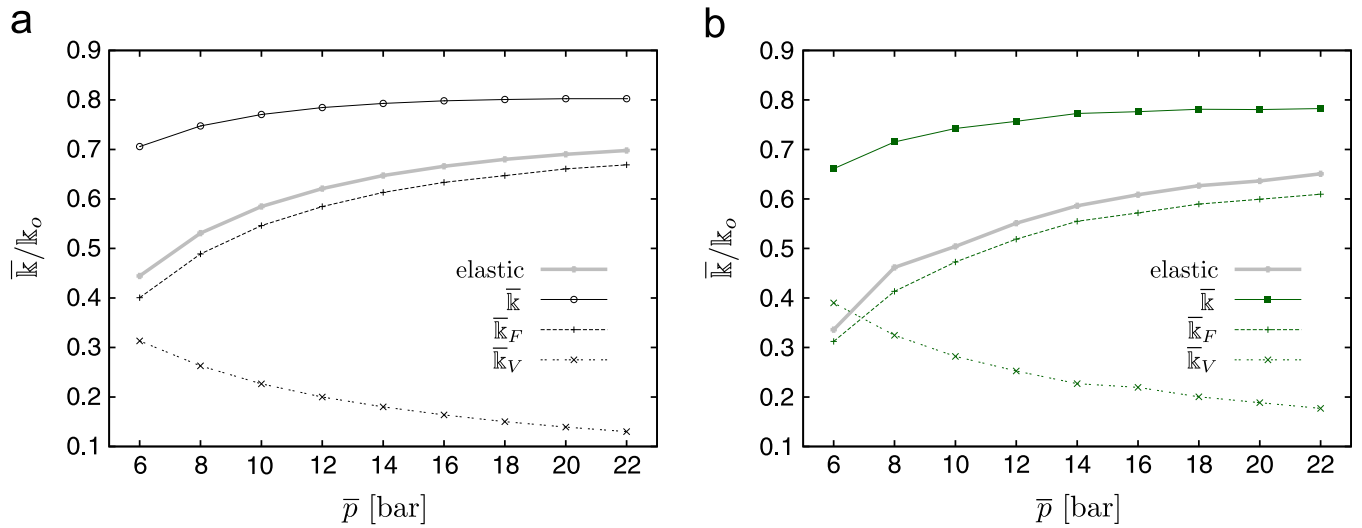

Fig. 7. For the circular and rectangular geometries of Fig. 3, the frictional $\left(\bar{k}_{F}\right)$ and viscous $\left(\bar{k}_{V}\right)$ contributions to $\mathbb{k}_{k}$ are monitored with varying $\bar{p}$. (a) Circular and (b) rectangular.
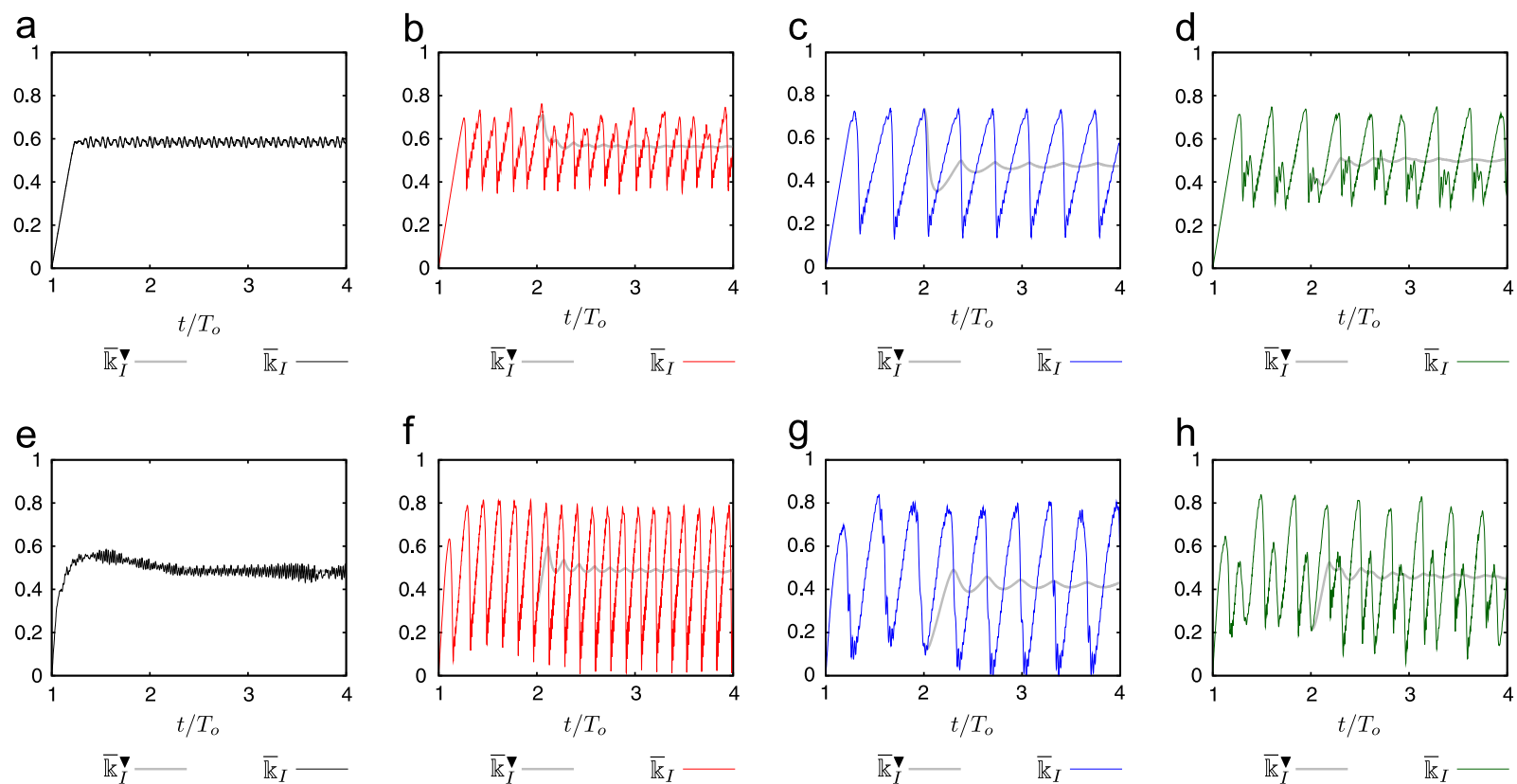

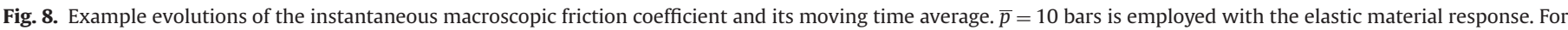

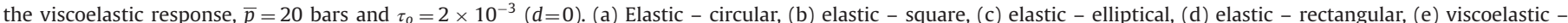
circular, (f) viscoelastic - square, $(\mathrm{g})$ viscoelastic - elliptical, and $(\mathrm{h})$ viscoelastic - rectangular.

causes the convergence to the full-relaxation limit to be delayed as the slip velocity is reduced. Since the aspect ratio is higher for the elliptical and rectangular particles, the frequency associated with the particle rolling is lower. Consequently, the two peaks of the viscous contribution approach and merge. The degree of merging is higher for a higher aspect ratio, in this case for the elliptical 
a

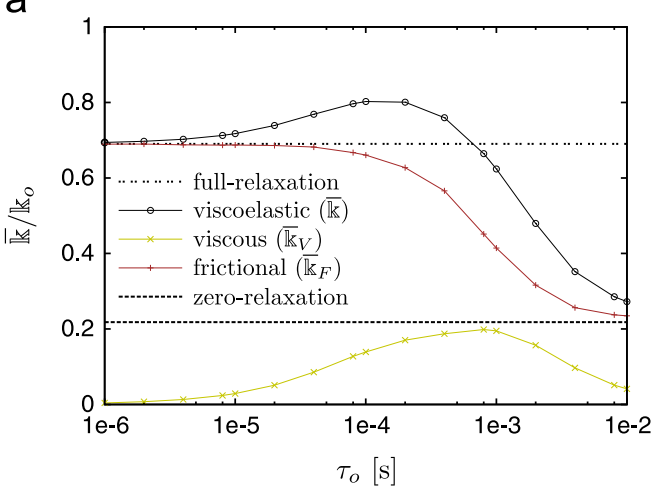

C

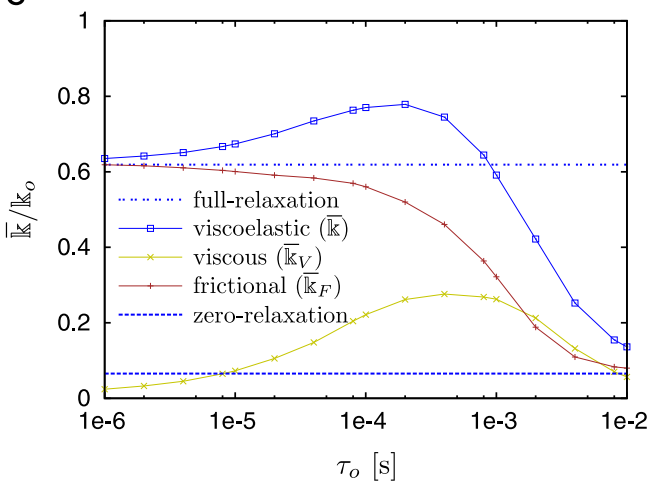

b

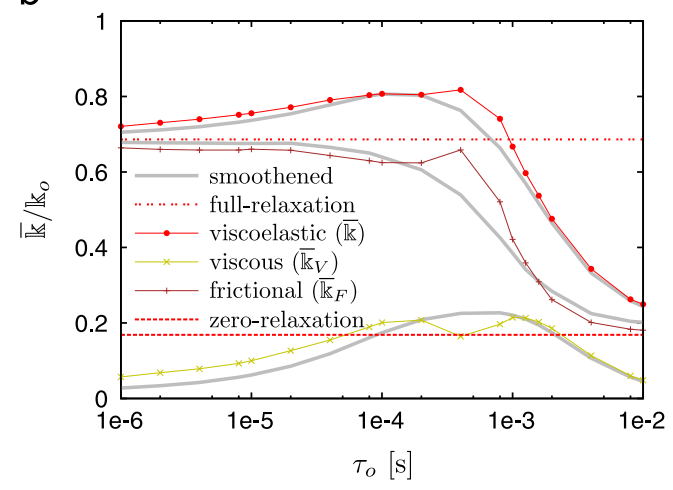

d

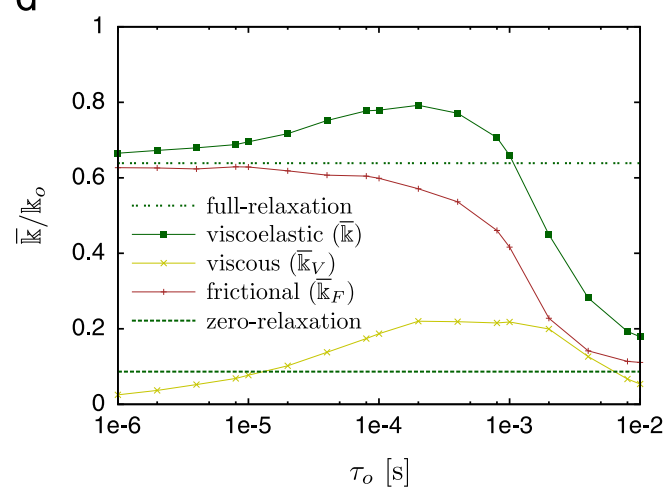

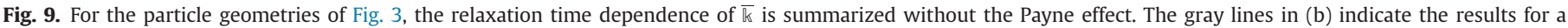
smoothened square with $p_{1}=p_{2}=3$ instead of 4 . (a) Circular, (b) square, (c) elliptical, and (d) rectangular.

geometry, so that a very smooth viscous contribution is observed. However, the delay in approaching the full-relaxation limit is persistent for all non-circular particles. Finally, it is remarked that the curves for the square particle can be significantly smoothened if the geometry edges are smoothened, for instance by choosing $p_{1}=p_{2}=3$ instead of 4 . The responses obtained with such a square-like geometry are shown on the background of Fig. 9 (b) with a gray line and are observed to be significantly different from the case with sharper edges. It is remarked that due to the relatively sharp edges of the square particle a much finer mesh resolution is needed in order to accurately capture the macroscopic frictional response, in particular at intermediate relaxation times. Presently, $N_{X}=80$ and $N_{Y}=25$ were employed - cf. Table 1.

While the equivalent relaxation time idea works well for circular particles, the additional excitation mechanism due to the rolling of non-circular particles may cause a deviation from this idealized behavior. Moreover, the Payne effect has a time scale associated with the reduction of the viscosity so that assessing its affect requires a direct control of the macroscopic slip velocity. For this purpose, the macroscopic slip velocity is varied with respect to its default value by one order of magnitude with and without the Payne effect and the results are compared with the corresponding ones from Fig. 9 in Table 5. The simulation time step size is varied, inversely proportional to the change in the slip velocity, for an accurate DEM-FEM interaction. Without the Payne effect, the circular particle displays the expected behavior. For noncircular particles, there is a satisfactory equivalence for low slip velocities although the degree of mismatch tends to increase with higher velocities. It is additionally observed that the presence of the Payne effect alters the frictional response drastically, as observed earlier in Fig. 6(b). In all cases, the Payne effect leads to a reduction of the relaxation time $\tau$ with respect to its default value $\tau_{0}$. The degree of reduction increases with the slip velocity directly through the velocity gradient magnitude through (15). The higher reduction at higher slip velocities delays the convergence to the zero-relaxation limit although little change is observed at low slip velocities. Consequently, the Payne effect is expected to not simply shift the $\overline{\mathbb{k}}$ curves in Fig. 9 to the right but rather distort them.

\subsection{Geometric bounds}

Consider a homogeneous elastic medium within which particles of irregular shape are dispersed. A well-known result from micromechanics states that the macroscopic stiffness associated with this heterogeneous material may be bounded by a fictitious construction wherein particles that geometrically bound the original ones are dispersed throughout the medium [46]. Motivated by this result, it is of interest to determine whether one may numerically bound the macroscopic frictional response from a complex interface particle geometry by computations that are based on simplified shapes which geometrically bound the original one. Among the examined particle geometries in earlier numerical examples, the circular one provides a geometric lower bound by construction. A geometric upper bound for the elliptical particle is a circle that has a radius equal to $1.5 r_{0}$. For the square particle, the bounding radius is approximately $\sqrt{2} r_{o} \approx 1.4 r_{o}$ and for the rectangular one it is approximately $1.6 r_{0}$. Since the one for the elliptical particle is close to these latter two cases, it is chosen to represent a geometric upper bound to all three particle geometries.

The response from an elastic boundary layer verifies that the responses from the two circular particles that geometrically bound the non-circular shapes deliver upper and lower bounds on the macroscopic frictional response - see Fig. 6(a). This is a significantly 
Table 5

The equivalent relaxation time is varied under $\tau_{0}$ - or $\bar{v}_{F}$-control, the latter with and without the Payne effect, and the measured $\overline{\mathbb{k}}$ is tabulated.

\begin{tabular}{|c|c|c|c|c|c|}
\hline Particle type & $\frac{\tau_{o} \times \bar{v}_{F}}{\left(10^{-4} \mathrm{~s}\right) \times(2.5 \mathrm{~m} / \mathrm{s})}$ & $\tau_{o}$ is varied $(d=0)$ & $\bar{v}_{F}$ is varied $(d=0)$ & $\bar{v}_{F}$ is varied $\left(d=10^{-5}\right)$ & $\bar{v}_{F}$ is varied $\left(d=10^{-6}\right)$ \\
\hline 1 (circular) & 0.1 & 0.359 & 0.359 & 0.346 & 0.351 \\
\hline 1 & 1 & 0.401 & 0.401 & 0.352 & 0.381 \\
\hline 1 & 10 & 0.312 & 0.312 & 0.388 & 0.383 \\
\hline 2 (square) & 0.1 & 0.382 & 0.390 & 0.371 & 0.396 \\
\hline 2 & 1 & 0.409 & 0.409 & 0.381 & 0.392 \\
\hline 2 & 10 & 0.418 & 0.374 & 0.382 & 0.412 \\
\hline 3 (elliptical) & 0.1 & 0.337 & 0.338 & 0.315 & 0.325 \\
\hline 3 & 1 & 0.385 & 0.385 & 0.329 & 0.364 \\
\hline 3 & 10 & 0.296 & 0.325 & 0.390 & 0.401 \\
\hline 4 (rectangular) & 0.1 & 0.348 & 0.350 & 0.327 & 0.338 \\
\hline 4 & 1 & 0.390 & 0.390 & 0.343 & 0.374 \\
\hline 4 & 10 & 0.330 & 0.327 & 0.386 & 0.405 \\
\hline
\end{tabular}

a

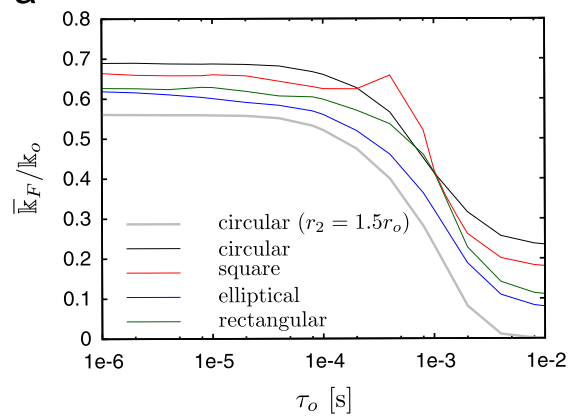

b

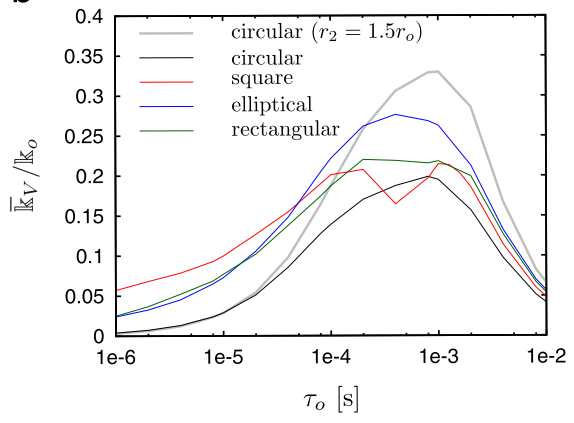

C

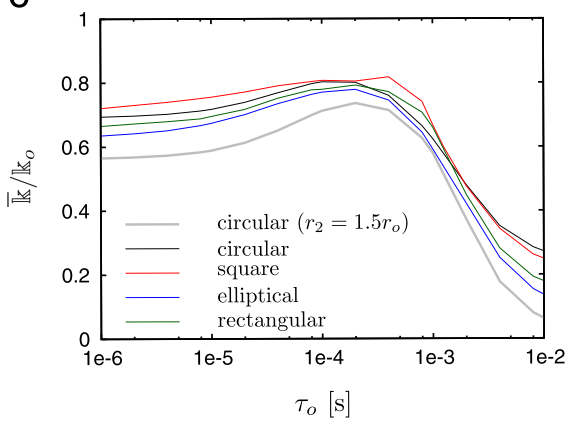

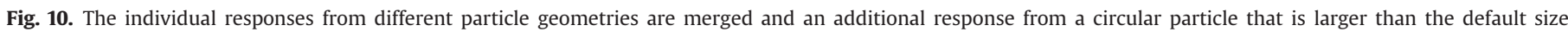
is considered. (a) Frictional contribution, (b) viscous contribution, and (c) total friction.

facilitating result since real particle geometries are difficult to represent. It appears, on the other hand, that this observation is limited to an elastic material response as in the original micromechanics result. Fig. 10 merges the frictional responses with a viscoelastic boundary layer from different particle geometries and additionally considers the response with the larger circular particle. While the frictional contributions are relatively well-bounded, the geometric bounds do not deliver bounds on the viscous contributions. In particular, they cannot capture the delay in the convergence to the full-relaxation limit since a circular particle displays not two but only a single frequency of excitation that is associated with its movement through the interface. Overall, however, the dominating contribution to the macroscopic response is frictional at low slip velocities and relaxation times so that the overall response is again relatively well-bounded. One can therefore conclude that bounding complex particle shapes by geometries that are easier to represent is an acceptable first step towards the characterization of the macroscopic frictional response.

\section{Conclusion}

In this work, the macroscopic frictional response of granular interfaces with non-circular particles was investigated. The microscopic system under consideration was modeled as a three-body contact problem wherein a rubber block is in contact with a surface that is partially covered by particles. The material model for rubber was based on a nonlinear viscoelasticity model that is enhanced with an amplitude-dependent relaxation behavior. The particle geometry was parametrized by convex superellipse shapes that require iterative contact detection algorithms as well as contact force computation schemes that are based on the discrete element method. Numerical investigations concentrated on isolating the effect of the particle geometry on the macroscopic frictional response, in particular with respect to the modification of the macroscopic non-Amontons and nonCoulomb type frictional behavior. In general, non-circular particles delivered a macroscopic response that is qualitatively similar to the response with a circular particle. This observation is primarily due to the fact that the particle displayed a rolling motion for all the test scenarios. However, a non-circular particle has a rolling resistance associated with it that causes sharp oscillations in the instantaneous macroscopic frictional response. The non-circular geometry also introduces an additional frequency of excitation so that the viscous contribution to the macroscopic frictional response is more spread across the relaxation spectrum of the viscoelastic material. Additionally, the results suggest that it may be possible to bound the macroscopic frictional response from a complex interface particle geometry by computations that are based on simplified shapes which geometrically bound the original one. While this observation offers a significant computational and experimental convenience towards replicating the granular media for the frictional characterization of the interface, the possibly significant effect of the underlying assumptions of (i) convex particle geometry, (ii) periodic particle distribution and (iii) a single particle layer must be assessed. It may readily be anticipated that multiple nonconvex particles coming into contact may strongly interlock, leading to a macroscopic response that cannot be bound by convex shapes even if there is a single layer. $n$ addition, generalizations towards a three-dimensional setting with improved rubber friction models are needed for qualitative and quantitative comparisons with experiments. Such investigations are currently being pursued. 


\section{Acknowledgment}

Partial support by the Michelin Tire Company is gratefully acknowledged.

\section{References}

[1] Gulseren O, Manini N, Meyer E, Tosatti E, Urbakh M, Vanossi A. New trends in nanotribology. Tribology Letters 2010;35(26-27):3455-82.

[2] Rabinowicz E. Friction and wear of materials. 2nd editionJohn Wiley \& Sons; 1995.

[3] Temizer İ, Wriggers P. A multiscale contact homogenization technique for the modeling of third bodies in the contact interface. Computer Methods in Applied Mechanics and Engineering 2008;198:377-96.

[4] Temizer I., Wriggers P. Inelastic analysis of granular interfaces via computational contact homogenization. International Journal for Numerical Methods in Engineering 2010;84:883-915.

[5] Pöschel T, Schwager T. Computational granular dynamics: models and algorithms. Berlin, Heidelberg, New York: Springer; 2005.

[6] Torquato S. Random heterogeneous materials: microstructure and macroscopic properties. Berlin, Heidelberg, New York: Springer; 2002.

[7] Schröder J, Balzani D, Brands D. Approximation of random microstructures by periodic statistically similar representative volume elements based on linealpath functions. Archive of Applied Mechanics 2011;81:975-97.

[8] Munjiza A. Combined finite-discrete element method.Chichester: Wiley; 2004

[9] Wriggers P. Computational contact mechanics. 2nd editionBerlin, Heidelberg, New York: Springer; 2006

[10] Laursen TA. Computational contact and impact mechanics. 1st edition (corr 2nd printing)Berlin, Heidelberg, New York: Springer; 2003.

[11] Hogue C. Shape representation and contact detection for discrete element simulations of arbitrary geometries. Engineering Computation 1998;15: 374-90.

[12] Houlsby GT. Potential particles: a method for modelling non-circular particles in DEM. Computers and Geotechnics 2009;36:953-9.

[13] Andrade JE, Lim K-W, Avila CF, Vlahinić I. Granular element method for computational granular mechanics. Computer Methods in Applied Mechanics and Engineering 2012:241-244:262-74.

[14] Wellmann C, Lillie C, Wriggers P. Comparison of the macroscopic behavior of granular materials modeled by different constitutive equations on the microscale. Finite Elements in Analysis and Design 2008;44:259-71.

[15] Zhou ZY, Pinson D, Zhoud RP, Yu AB. Discrete particle simulation of gas fluidization of ellipsoidal particles. Chemical Engineering Science 2011;66:6128-1545.

[16] Wellmann C. A two-scale model of granular materials using a coupled DE-FE approach. PhD thesis, Leibniz Universität Hannover, Hannover, Germany; 2011

[17] Renouf M, Massi F, Fillot N, Saulot A. Numerical tribology of a dry contact. Tribology International 2011:44:834-44.

[18] Cao H-P, Renouf M, Dubois F, Berthier Y. Coupling continuous and discontinuous descriptions to model first body deformation in third body flows. Journal of Tribology 2011;133:041601.

[19] Österle W, Dmitriev AI, Kloß H. Possible impacts of third body nano structure on friction performance during dry sliding determined by computer simulation based on the method of movable cellular automata. Journal of Tribology 2012:48:128-36.

[20] Iordanoff I, Khonsari MM. Granular lubrication: toward an understanding of the transition between kinetic and quasi-fluid regime. Journal of Tribology 2004; $126: 137-45$.

[21] Jang JY, Khonsari MM. On the granular lubrication theory. Proceedings of the Royal Society A: Mathematical, Physical and Engineering Sciences 2005;461: 3255-78.
[22] Renouf M, Cao H-P, Nhu V-H. Multiphysical modeling of third-body rheology. Tribology International 2011;44:417-25.

[23] Andrade JE, Avila CF, Hall SA, Lenoir N, Viggiani G. Multiscale modeling and characterization of granular matter: from grain kinematics to continuum mechanics. Journal of the Mechanics and Physics of Solids 2011;59:237-50.

[24] Wellmann C, Wriggers P. A two-scale model of granular materials. Computer Methods in Applied Mechanics and Engineering 2012;205-208:46-58.

[25] Persson BNJ. Sliding friction. 2nd editionBerlin, Heidelberg, New York: Springer-Verlag; 2000

[26] Schallamach A. The velocity and temperature dependence of rubber friction. Proceedings of the Physical Society: Section B 1953;66:386-92.

[27] Bartenev GM, Lavrent'ev VV, Konstantinova NA. Temperature dependence of the actual contact area and friction force of high-elasticity materials. Polymer Mechanics 1967;3:726-9.

[28] Grosch KA. The relation between the friction and visco-elastic properties of rubber. Proceedings of the Royal Society of London. Series A. Mathematical, Physical and Engineering Sciences 1963;274:21-39.

[29] Vorvolakos K, Chaudhury MK. The effects of molecular weight and temperature on the kinetic friction of silicone rubbers. Langmuir 2003;19:6778-87.

[30] Klüppel M, Heinrich G. Rubber friction on self-affine road tracks. Rubber Chemistry and Technology 2000;73:578-606.

[31] Persson BNJ. Theory of rubber friction and contact mechanics. Journal of Chemical Physics 2001;115:3840-61.

[32] Le Gal A, Klüppel M. Investigation and modelling of rubber stationary friction on rough surfaces. Journal of Physics: Condensed Matter 2008;20:015007.

[33] Carbone G, Lorenz B, Persson BNJ, Wohlers A. Contact mechanics and rubber friction for randomly rough surfaces with anisotropic statistical properties. European Physics Journal E: Soft Matter and Biological Physics 2009;29: 275-84.

[34] Wriggers P, Reinelt J. Multi-scale approach for frictional contact of elastomers on rough rigid surfaces. Computer Methods in Applied Mechanics and Engineering 2009;198:1996-2008.

[35] Carbone G, Putignano C. A novel methodology to predict sliding and rolling friction of viscoelastic materials: theory and experiments. Journal of the Mechanics and Physics of Solids 2013;68:1822-34.

[36] Timma K, Myant C, Spikes HA, Grunze M. Particulate lubricants in cosmetic applications. Tribology International 2011:44:1695-703.

[37] Vilt SG, Martin N, McCabe C, Jennings GK. Frictional performance of silica microspheres. Tribology International 2011;44:180-6.

[38] Marckmann G, Verron E. Comparison of hyperelastic models for rubber-like materials. Rubber Chemistry and Technology 2006;79(5):835-58.

[39] Puso MA, Laursen TA. A mortar segment-to-segment frictional contact method for large deformations. Computer Methods in Applied Mechanics and Engineering 2004;193:4891-913.

[40] Hüeber S, Stadler G, Wohlmuth BI. A primal-dual active set algorithm for three-dimensional contact problems with Coulomb friction. SIAM Journal on Scientific Computing 2008;30:572-96.

[41] Tur M, Fuenmayor FJ, Wriggers P. A mortar-based frictional contact formulation for large deformations using Lagrange multipliers. Computer Methods in Applied Mechanics and Engineering 2009;198:2860-73.

[42] Temizer İ. A mixed formulation of mortar-based contact with friction. Computer Methods in Applied Mechanics and Engineering 2013;255:183-95.

[43] Schwager T, Pöschel T. Coefficient of restitution for viscoelastic spheres: the effect of delayed recovery. Physical Review E 2008;89:051304.

[44] Reese S, Govindjee S. A theory of finite viscoelasticity and numerical aspects. International Journal of Solids and Structures 1998:35(26-27):3455-82.

[45] Rendek M, Lion A. Amplitude dependence of filler-reinforced rubber: experiments, constitutive modelling and FEM - implementation. International Journal of Solids and Structures 2010;47:2918-36.

[46] Hill R. Elastic properties of reinforced solids: some theoretical principles. Journal of the Mechanics and Physics of Solids 1963;11:357-72. 